\title{
Dynamic Characteristics of a Four-Bar Linkage with a Composite Coupler
}

\author{
Raghu Echempati ${ }^{\dagger}$ \\ Department of Mechanical Engineering, Kettering University, Flint, MI 48504, USA
}

(Received 26 January 2004; accepted 28 July 2004)

\begin{abstract}
Most modern machinery and automated industrial mechanical equipment operates at high speeds to attain high productivity. Damping plays a prominent role in controlling vibration in structural members of these mechanical systems. Depending upon the specific application and the type of desired dynamic properties, different engineering materials with improved vibrational and damping characteristics are being used by many researchers and by several industrial manufacturers. A four-bar mechanism is one such application that must have the basic characteristics, such as low inertia, high strength, and stiffness, to enable smooth power transmission without excessive vibrations and deflections. In order to study the overall dynamic performance of four bar mechanisms, in this research, it is proposed that the isotropic metal members be replaced by composite materials. The work presented in this paper deals with an experimental study of the dynamic response of the coupler link of four-bar linkage mechanisms fabricated from different hybrid glass-graphite/epoxy pultruded composite beams. The response characteristics include the determination of midpoint strain, the system damping, and the fundamental frequency of the couplers. These characteristics are compared with coupler links made out of steel and aluminum. This study is very useful in exploring whether pultruded composites can potentially be used in high-speed industrial mechanism devices.
\end{abstract}

${ }^{\dagger}$ Member of the International Institute of Acoustics and Vibration (IIAV)

\section{INTRODUCTION}

Most industrial machinery consists of one or more mechanisms to perform different tasks. During the last 20 years, much research has been conducted towards understanding the mechanics of composites and their applications in industry. Composites are replacing traditional metals because of their high strength-to-weight ratio and good damping characteristics. Designers in the analysis of machinery and mechanisms have always been challenged to research the industrial applications of composites. Thus, the intent of this paper is to study the dynamic characteristics of the coupler member of a mechanism rather than investigating the dynamic characterisation of the composites.

Much research has been conducted on the dynamic response, balancing, and optimisation studies of mechanisms that use steel or aluminum alloys. For example see, the work of Imam et al. and Berkof and Lowen., ${ }^{1,2}$ A great deal of research has also been done on the dynamic characteristics of flexible mechanisms. Erdman and Sandor used the finite element method to determine the response of flexible four bar mechanism links made of steel. ${ }^{3}$ Alexander and Lawrence experimentally studied the strain histories of the coupler and rocker midpoints of a flexible four-bar mechanism made of aluminum. ${ }^{4}$ In their work, the cyclic bending strain variations, frequency response curves, and damping estimates were presented, and the results were compared with their analytical results. Sadler and Sandor studied the elastodynamic response of mechanisms by using the lumped parameter method and compared their results with those of Alexander and Lawrence. ${ }^{5}$

In Part I of their paper, Jandrasits and Lowen analysed the transverse elastic behaviour of a counterweighted steel rocker link with an end mass. ${ }^{6}$ The Hamilton's integral and the method of Kantorovich were used to obtain the linearised decoupled Hill's equation, which was solved to furnish the time portion of the solution. The space portion of the solution was determined using normal modes of free vibration of the complex link. In Part II of their paper, the analytical techniques developed in Part I were applied to an example mechanism, and the results were compared with those of an associated experiment.

Lowen and Chassapis gave an update of literature surveys in the field of elastic behaviour of linkages during the period from 1977 to $1983 .{ }^{7}$ Based on their work, Balasubramonian and Echempati derived the equation of motion for the transverse vibration of a counterweighted elastic coupler of a four-bar linkage by using the energy method. ${ }^{8}$ In their modelling, the axial foreshortening effect of time dependent axial force was also included. Responses at various speeds were presented, and some of them were compared with those of the existing literature.

In all of the investigations mentioned above, the mechanisms studied were fabricated from steel or aluminum alloys. As mentioned before and in order to obtain an optimum lightweight design, it was proposed by many researchers to fabricate the members out of fibre-reinforced materials such as graphite or boron composites. Jones and Dharan quoted that many automotive or aerospace components have been fabricated from composite materials with weight savings of 70 percent compared to designs that use high strength steels., ${ }^{\mathbf{9} 10}$ As reported by Paipetis and Groontenhuis, composite materials, depending up on the type of fibre and fibre orientation, generally possess good vibrational characteristics and inherent material damping which can be up to thirty times greater than that of aluminum. ${ }^{11}$ Unidirectional, plastic composite materials made of glass-graphite/epoxy materials are gaining importance because of their excellent vibration and damping 